\title{
Efficient and innocuous delivery of small interfering RNA to microglia using an amphiphilic dendrimer nanovector
}

\author{
Aleksandra Ellert-Miklaszewska*,1, Natalia Ochocka1, Marta Maleszewska1 , Ling Ding², \\ Erik Laurini ${ }^{3}$, Yifan Jiang ${ }^{2}$, Adria-Jaume Roura1 ${ }^{1}$ Suzanne Giorgio², Bartlomiej Gielniewski ${ }^{1}$, \\ Sabrina Pricl ${ }^{3}$, Ling Peng**,2 (iD) \& Bozena Kaminska ${ }^{1}$ \\ ${ }^{1}$ Laboratory of Molecular Neurobiology, Neurobiology Center, Nencki Institute of Experimental Biology of the Polish Academy of \\ Sciences, Warsaw, 02-093, Poland \\ ${ }^{2}$ Aix-Marseille Université, CNRS, Center Interdisciplinaire de Nanoscience de Marseille, UMR 7325, Equipe Labellisée Ligue Contre \\ le Cancer, 13288, Marseille, France \\ ${ }^{3}$ Molecular Biology \& Nanotechnology Laboratory (MolBNL@UniTS), Department of Engineering \& Architecture, University of \\ Trieste UNITS, 34127, Trieste, Italy \\ *Author for correspondence: a.ellert@nencki.gov.pl \\ **Author for correspondence: ling.peng@univ-amu.fr
}

Aim: Alterations of microglia, the brain-resident macrophages, are associated with numerous brain pathologies. Genetic manipulation of microglia in diseases using small interfering RNA (siRNA) is hampered by the lack of safe and efficient siRNA delivery methods. We assessed the amphiphilic dendrimer (AD) for functional siRNA delivery and gene knockdown in primary microglia. Materials \& methods: We characterized the ability of AD to form nanoparticles with siRNA, and studied their size, surface potential, cell uptake and gene silencing in rodent microglia. Results: AD effectively delivered siRNA to primary microglia and decreased target gene and protein expression, leading to transcriptomic changes without affecting basal microglial functions. Conclusion: The dendrimer AD promises to be an innocuous carrier for siRNA delivery into microglia.

\section{Graphical abstract:}

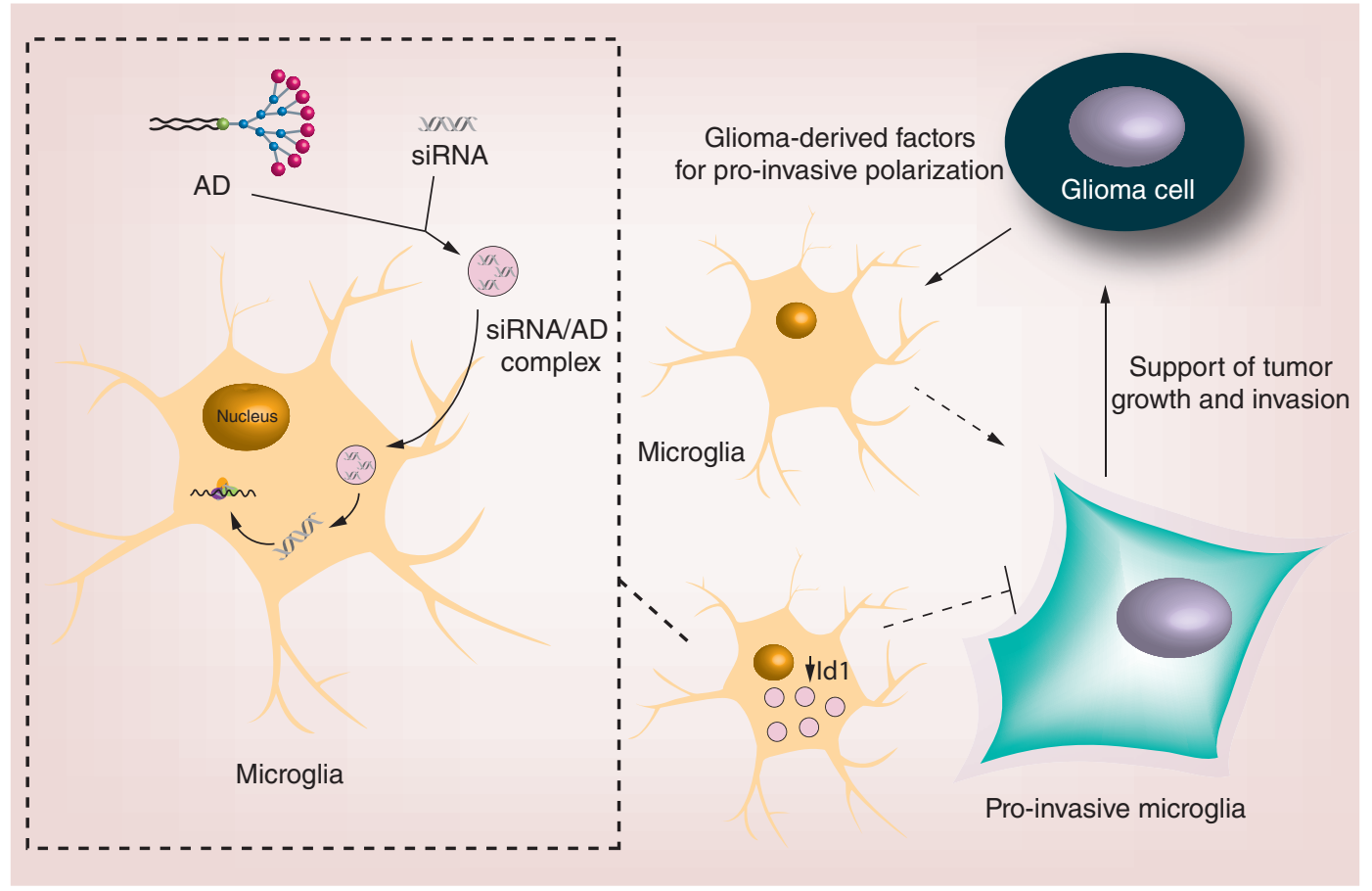

Future Medicine 
First draft submitted: 30 April 2019; Accepted for publication: 17 June 2019; Published online: 28 August 2019

Keywords: amphiphilic dendrimer $\bullet$ basal microglial responses $\bullet$ dendrimer nanovector $\bullet$ gene silencing $\bullet$ gliomainitiated response $\bullet$ inhibitor of differentiation Id $1 \bullet$ microglial function $\bullet$ nonviral vector $\bullet$ primary microglia $\bullet$ siRNA delivery

Microglia are the resident macrophages of the central nervous system (CNS). They participate in brain development, regulation of homeostasis and synaptic plasticity, as well as protecting the brain from infections, metabolic disturbances or misfolded proteins [1-3]. However, aberrant or chronic microglial activation leads to neuroinflammatory brain damage linked to the pathogenesis of stroke and many neurodegenerative and psychiatric disorders [4-9]. Moreover, microglia contribute to the progression of brain tumors, including the most common and deadliest glioblastoma multiforme (GBM) [10]. Microglia and peripheral macrophages massively infiltrate GBM tumors [11], where they become polarized to promote glioma invasion, immunosuppression and angiogenesis $[12,13]$. Due to their critical role as instigators of inflammatory or pro-tumorigenic events, microglia are considered to be a potentially promising therapeutic target [14]. Therefore, further molecular insights into microglial functions are of paramount importance for our understanding of neurological disorders and for fostering the development of effective treatments for the related diseases.

Genetic manipulation using small interfering RNA (siRNA) is frequently used for studying genotype-phenotype relationships and identifying potential drug targets and candidates [15-17]. RNA interference (RNAi) is a biological process in which siRNA molecules specifically inhibit gene translation by neutralizing targeted mRNA molecules via Watson-Crick base-pairing. However, siRNA delivery to microglia has been difficult due to the high immunereactivity of microglia to transfection agents and the characteristic high charge and enzyme sensitivity of siRNA molecules [18,19]. Although both viral and nonviral vectors have been established for nucleic acid delivery, neither class is safe for siRNA delivery to primary microglia. Viral vectors are highly efficient for delivering siRNA via a short hairpin RNA (shRNA)-coding sequence, but they carry a high risk of mutagenesis and upregulation of pro-inflammatory factors, which interfere with experimental conditions in studies on immune system cells. Certain nonviral lipid and polymer vectors perform well for the majority of established cell lines, including immortalized mouse microglial BV2 cells [20], but they have marginal efficacy; moreover, they also induce inflammatory gene expression and have considerable toxic effects on primary microglia cultures. It is worth noting that microgliaderived cell lines, frequently used for mechanistic studies, may acquire, during immortalization and repeated passaging, different features that are not present under physiological conditions in primary cells [21]. Although there are a few reports of nanoparticle-enhanced delivery of nucleic acids to peritoneal macrophages [22] or macrophagelike J774 cells [23], gene expression was not analyzed in those studies and the effects on macrophage functions are unknown. Therefore, there is an urgent need for safe and effective vectors, which do not induce undesired microglial activation - for siRNA delivery to primary microglia - for not only therapeutic purposes but also a deeper understanding of the role of microglia and macrophages in human brain pathologies.

Dendrimers, a special family of highly branched molecules with well-defined architecture and nanoscale size, represent a promising platform for siRNA and drug delivery [24-28]. The advantages of dendrimer nanocarriers over other delivery vectors are the precisely controlled and ramified molecular structure, high cargo payload and the abundant peripheral groups, which allow cooperative multivalence and fine-tuning of the dendrimer properties for effective on-demand delivery [29,30]. Poly(amidoamine) (PAMAM) dendrimers are the most intensively studied dendrimers for siRNA delivery [24,29]. PAMAM dendrimers have primary amine terminals, which are positively charged at physiological $\mathrm{pH}$, so that they can form stable complexes with negatively charged siRNA; thus, masking the charges of siRNA and protecting it from degradation [31]. In addition, PAMAM dendrimers harbor tertiary amines at the interior, which act as a proton sponge at lower $\mathrm{pH}$ (i.e., $\mathrm{pH}$ 5), and promote endosome escape; hence, facilitating nucleic acid release [32]. Recently, amphiphilic PAMAM dendrimer nanosystems exploiting the delivery advantages of both lipid and dendrimer vectors have been established by our group [28,33-38]. These dendrimer delivery systems produced specific and long-term gene silencing in vitro and in vivo in various disease models, outperforming commercial nonviral vectors [24]. Now, we present and discuss novel results obtained with the amphiphilic dendrimer (AD), which bears two hydrophobic chains and one hydrophilic PAMAM dendron for effective siRNA delivery to microglia in primary cell cultures (Figure 1). Notably, this AD dendrimer formed small and robust nanoparticles with siRNA, which prevented siRNA degradation and facilitated siRNA uptake 
(A)

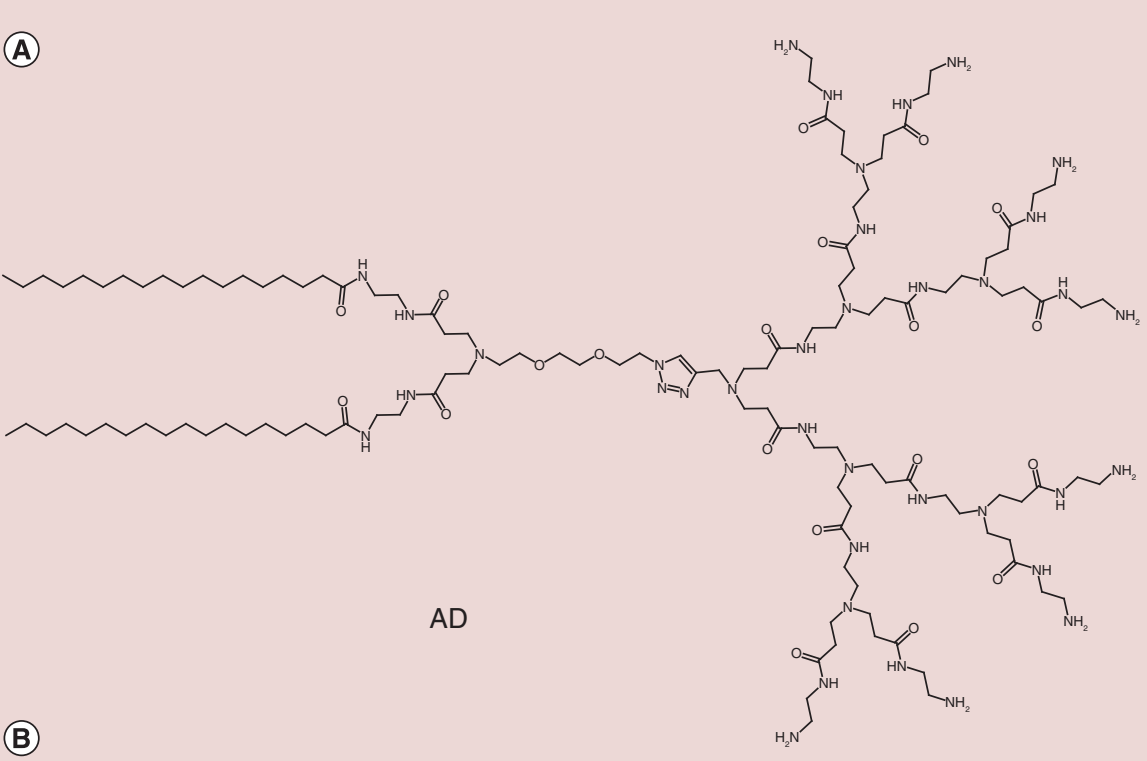

(B)

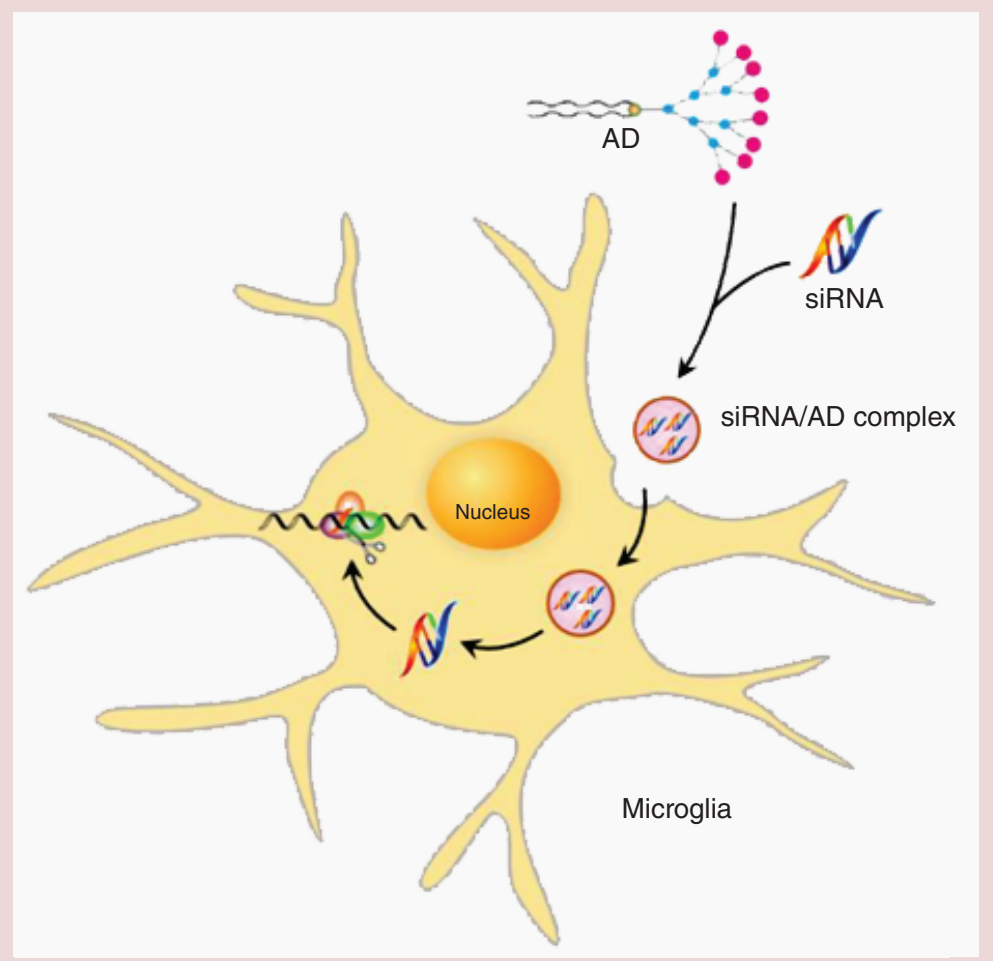

Figure 1. Amphiphilic dendrimer - a new carrier for siRNA delivery to microglia. (A) Chemical structure of the amphiphilic poly(amidoamine)dendrimer. (B) Schematic presentation of siRNA/AD complex formation and AD-mediated siRNA delivery.

AD: Amphiphilic dendrimer.

into microglia. The subsequent siRNA-mediated gene silencing at the mRNA and protein levels had consequential biological effects, in other words, attenuation of the pro-invasive polarization of microglia stimulated by glioma tumor cells. Remarkably, this dendrimer nanovector did not induce unwanted phenotype, or transcriptome alterations, or cellular toxicity in the microglia. To the best of our knowledge, this is the first-ever reported vector that effectively delivers functional siRNA to primary microglia cultures without affecting the basal microglial functions 
and responses. Consequently, it constitutes a promising tool for siRNA delivery in microglia and provides a new outlook for functional and therapeutic studies in various models of CNS diseases.

\section{Methods}

Materials

The $\mathrm{AD}$, synthesized according to our previously reported protocol [36], was dissolved in milli-Q water to $630 \mu \mathrm{M}$ as the stock solution. The control siRNA (ON-TARGET plus non-targeting pool; siCtrl), the siRNA targeting Id1 (ON-TARGET plus SMART pool; siId1), the red fluorescent tag DY-547 labeled RISC, independent siRNA transfection indicator (siGlo) and the red fluorescent-tag DY-547 labeled nontargeting control siRNA (siCtrl-Glo) were obtained from Dharmacon, Inc. (CO, USA).

\section{Agarose gel retardation analysis}

The siRNA was dissolved in diethylpyrocarbonate (DEPC)-treated water at an appropriate concentration and stored at $4.0^{\circ} \mathrm{C}$. The dendrimer $\mathrm{AD}$ and siRNA solutions were mixed at various $\mathrm{N} / \mathrm{P}$ ratios $(=$ [total terminal amines in the dendrimer $\mathrm{AD}$ ]/[total phosphates in the siRNA]) from $2.5: 1$ to $15: 1$, and incubated at $37^{\circ} \mathrm{C}$ for $30 \mathrm{~min}$. The siRNA/AD complexes were analyzed using electrophoretic mobility-shift assay $(1.2 \%$ agarose gel in standard Tris-Borate-EDTA [TBE] buffer), with naked siRNA as a control. The final concentration of siRNA was adjusted to $80 \mathrm{ng} /$ well. The siRNA bands were stained by ethidium bromide and detected by a Herolab EASY CCD camera (type 429K; Herolab, Wiesloch, Germany).

\section{Transmission electron microscopy}

Size and morphology of the siRNA/AD complexes were analyzed by transmission electron microscopy (TEM) using a JEOL3010 transmission electron microscope (Tokyo, Japan). A solution $(10 \mu \mathrm{l})$ of siRNA $(3.0 \mu \mathrm{M})$ was mixed with a solution $(10 \mu \mathrm{l})$ of dendrimer $\mathrm{AD}$ in milli-Q water at $\mathrm{N} / \mathrm{P}$ ratio of 10 and incubated for $30 \mathrm{~min}$ at $37^{\circ} \mathrm{C}$. The mixture was then diluted 20 -fold, an aliquot of $4.0 \mu \mathrm{l}$ was withdrawn, deposited onto a standard carbon-coated copper TEM grid and dried in air for $60 \mathrm{~min}$ at $37^{\circ} \mathrm{C}$. The grid was stained with $3.0 \mu \mathrm{l}$ uranyl acetate $(2.0 \%$ in aqueous solution) for $5.0 \mathrm{~s}$, and the excess uranyl acetate was removed by filter paper. The dried specimens were observed at an accelerating voltage of $300 \mathrm{kV}$. Data were analyzed using the Digital Micrograph software

\section{Dynamic light scattering analysis}

The size, size distribution and zeta potential of the siRNA/AD complexes were determined using dynamic light scattering (DLS). The proper amount of siRNA and $\mathrm{AD}$ solutions $(\mathrm{N} / \mathrm{P}$ ratio $=10$, final siRNA concentration $=1.5 \mu \mathrm{M}$ ) were mixed, and incubated at $37^{\circ} \mathrm{C}$ for $30 \mathrm{~min}$. This freshly prepared solution was measured using a Zetasizer Nano ZS (Malvern Ltd., Malvern, UK) equipped with a standard He-Ne ion laser of $633 \mathrm{~nm}$ at $25^{\circ} \mathrm{C}$. All experiments were performed in triplicate.

\section{RNase A protection assay}

Aliquots of siRNA and $\mathrm{AD}$ solutions were mixed at $\mathrm{N} / \mathrm{P}$ ratio $=10$, and kept at $37^{\circ} \mathrm{C}$ for $30 \mathrm{~min}$. The obtained siRNA/AD complexes were incubated in the presence of $0.25 \mu \mathrm{g} / \mu \mathrm{l}$ RNase A at $37^{\circ} \mathrm{C}$ for different periods of time and then treated with $1.0 \%$ SDS solution at $4.0^{\circ} \mathrm{C}$ for siRNA release. Samples were run in a $1.2 \%$ agarose gel containing ethidium bromide and then detected by a Herolab EASY CCD camera (type 429K; Herolab, Wiesloch, Germany). Naked siRNA served as a control.

\section{Isothermal titration calorimetry measurements}

Isothermal titration calorimetry (ITC) experiments were conducted using a MicroCal PEAQ-ITC calorimeter (Malvern Ltd.) at $25^{\circ} \mathrm{C}$. The cell volume was $208 \mu \mathrm{l}$. All experiments were conducted by step-by-step injections of a constant volume of siRNA solution into the calorimetric cell containing a solution of $\mathrm{AD}$ in ultrapure water. Specifically, the siRNA solution $(25 \mu \mathrm{M})$ was injected in 19 portions of $2.0 \mu \mathrm{l}$ at 600 -s intervals, while the concentration of $\mathrm{AD}$ in the calorimeter cell was $50 \mu \mathrm{M}$. All solutions were degassed for $30 \mathrm{~min}$ at room temperature under stirring at 600 r.p.m. prior to each experiment. After washing, the cell was prerinsed with a portion of the ultrapure water solution and upon filling the cell and syringe, stirring was turned on and each system was allowed to thermally equilibrate for $30 \mathrm{~min}$. The heat signals resulting from mixing, dilution effects and liquid friction were 
further confirmed by control experiments (data not shown); accordingly, they were subtracted from the relevant datasets to yield the corrected integrated data. All experiments were run in triplicate.

\section{Cell culture \& transfection}

Microglial primary cultures were prepared from the cerebral cortices of P0-P2 Wistar rat pups and C57BL6J mouse pups as previously described [39,40]. Microglial cells were isolated from glial culture monolayers by gentle shaking, then collected by centrifugation, checked for viability and seeded at a density of $2-3 \times 10^{5} \mathrm{cells} / \mathrm{cm}^{2}$ onto plastic dishes (for nonadherent cultures) or glass culture slides in high-glucose culture medium (DMEM supplemented with Glutamax, 10\% fetal bovine serum [Gibco, Invitrogen; CA, USA] and 100 units $/ \mathrm{ml}$ of penicillin, $100 \mu \mathrm{g} / \mathrm{ml}$ of streptomycin). Microglial cultures were used for experiments $48 \mathrm{~h}$ after seeding. Cells were stimulated with $100 \mathrm{ng} / \mathrm{ml}$ lipopolysaccharide from Salmonella enteritidis (Sigma-Aldrich, MO, USA) or conditioned media from rat C6 glioma cells (ATCC, VA, USA). Glioma conditioned media (GCM) were prepared as follows: $24 \mathrm{~h}$ after seeding $1 \times 10^{6} \mathrm{C} 6$ cells onto 100-mm dishes, standard culture media were exchanged for $8 \mathrm{ml}$ of the medium used for microglial cultures. Conditioned media were harvested after $24 \mathrm{~h}$, centrifuged at $300 \times \mathrm{g}$ for $10 \mathrm{~min}$ and added to microglial cultures. For lentiviral infection, $1 \times 10^{3}$ viral particles per cell were added to microglia cultures. Lentiviral vectors were prepared at the Laboratory of Cell Engineering, Nencki Institute of Experimental Biology and expressed shRNA targeting Firefly Luciferase with no significant homology to any mammalian transcript. For siRNA delivery using Viromer, microglia cells were transfected with $25 \mathrm{nM}$ siGlo or control nontargeting siRNA using Viromer blue transfection reagent (Lipocalyx) at $0.1 \%$ dilution in culture medium. After $4 \mathrm{~h}$ the transfection medium was removed and cells were kept under standard conditions for 24 or $48 \mathrm{~h}$. For siRNA delivery using dendrimer AD, microglial cells were treated with siRNA/dendrimer AD complexes prepared in OptiMEM and added at 1:10 dilution to standard culture medium. Final siRNA concentrations in the cell culture were 12.5 to $50 \mathrm{nM}$ complexed with dendrimer $\mathrm{AD}$ at $\mathrm{N} / \mathrm{P}=10$. The effects of transfection procedure on gene and protein expression, cell viability and proliferation were analyzed at indicated time points.

\section{Immunocytochemistry}

To visualize endosomes, the cells were stained for EEA1. Microglia cells cultured on coverslips were fixed with $2 \%$ paraformaldehyde at indicated time points postdelivery of siRNA/AD complexes, permeabilized and treated with free-aldehyde quenching solution followed by a blocking solution. The cells were then stained with rabbit anti-EEA1 antibody (1:500, Enzo, NY, USA), followed by AlexaFluor488-conjugated donkey anti-rabbit antibody (1:800, Invitrogen, USA). The cell nuclei were labeled with DAPI (1:1000, Sigma) and finally the coverslips were mounted with Fluorescence Mounting Medium (Dako North America Inc., CA, USA) on glass slides. Subcellular localization of siRNA/AD complexes was verified using z-stack optical sections obtained with a confocal microscope (Fluoview FV10i, Olympus Corp., Tokyo, Japan). Representative orthogonal views were prepared from at least 12 z-stack optical sections using FV10-ASW software (Olympus Corp.).

\section{Reverse transcription PCR}

Total RNA was isolated using RNeasy kits (Qiagen, Hilden, Germany) and used as a template to synthesize cDNA by extension of oligo(dT) 15 primers $(2.5 \mathrm{mmol} / \mathrm{l})$ with $200 \mathrm{U}$ of SuperScritpIII reverse transcriptase (Life Technologies, CA, USA). Amplifications were performed in a $10-\mu l$ reaction volume containing SYBR PCR MasterMix (Life Technologies) and a set of primers (Supplementary Table 1). The amount of target mRNA was first normalized to the expression level of the $18 \mathrm{~S}$ rRNA amplified from the same sample and then to untreated controls. Data were analyzed with the Relative Quantification $\left({ }^{\Delta \Delta} \mathrm{Ct}\right)$ method using QuantStudio 12K Flex software (Life Technologies).

\section{The mRNA library preparation \& RNA sequencing}

Integrity and quality of RNA were assessed with an Agilent 2100 Bioanalyzer using an RNA 6000 Nano Kit (Agilent Technologies, CA, USA). In total, six strand-specific RNA libraries for sequencing were prepared (three biological replicates/treatment) using a KAPA Stranded mRNA-Seq Kit (Kapa Biosystems, MA, USA). Poly-A mRNAs were purified from $200 \mathrm{ng}$ of total RNA using poly-T-oligo-magnetic beads (Kapa Biosystems). The mRNAs were fragmented and first-strand cDNA was synthesized using reverse transcriptase and random hexamers. Second-strand cDNA synthesis was performed by removing the RNA templates and synthesizing replacement strands, incorporating dUTP in place of dTTP to generate double-stranded (ds) cDNA. The dsDNA fragments 


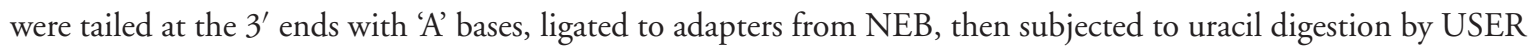
enzyme (NEB, MA, USA). Amplification of fragments with adapters ligated on both ends was performed by PCR using primers containing TruSeq barcodes (NEB). Final libraries were analyzed using Bioanalyzer and Agilent DNA High Sensitivity chips (Agilent Technologies) to confirm fragment sizes ( 300-350 bp). Quantification was performed with a Quantus fluorometer and QuantiFluor dsDNA System (Promega, WI, USA). Libraries were loaded at a concentration of $7.5 \mathrm{pM}$ onto a rapid run flow cell and were paired-end sequenced on an Illumina HiSeq 1500.

\section{Bioinformatics analysis}

RNA-seq reads were trimmed and aligned to the rat genome (version rn6) using the Trimmomatic [41] and STAR software [42]. Samples were aligned to the transcriptome using the Rattus norvegicus gene annotation file from Ensembl and reads were counted by genes using the featureCounts program [43]. Data quality analysis and batch effect removal was performed using the NOIseq package [44] followed by differential analysis in order to compare the siCtrl/AD- and siId1/AD-treated microglia cultures, both of which were stimulated for $6 \mathrm{~h}$ with GCM.

Datasets were analyzed for differences in individual gene expression (magnitude of fold change, FC), as well as for functional pathway enrichment analysis using the Kyoto Encyclopedia of Genes and Genomes (KEGG) database. Identification of functional groups of genes was performed using the clusterProfiler package [45]. Genes that were differentially expressed in terms of their false discovery rate (FDR) corrected p-values were sorted according to their FC values; separate analyses for down- and upregulated genes were performed on genes with counts per million $(\mathrm{CPM})>3$.

\section{Western blotting}

Protein extraction and western blotting were performed as reported in [46] with specific antibodies (all 1:1000) recognizing Id1 (B-8; sc-133104, Santa Cruz Biotechnology) and recognizing Id3 (D16D10; \#9837), cMyc (D84C12; \#5605) and the phosphorylated Rb (Ser807/811; \#9308), all purchased from Cell Signaling (MA, USA). Horseradish peroxidase-conjugated anti-rabbit IgG and anti-mouse IgG antibodies (1:10000) were from Vector Laboratories, Inc., (CA, USA). Immunocomplexes were visualized using ECL (Amersham, Germany). The membranes were stripped and re-probed with anti-GAPDH antibody (1:25000, \#MAB374 EMD Millipore Corp., MA, USA). The molecular weight of proteins was estimated with prestained protein markers (Thermo Fisher Scientific, Vilnius, Lithuania).

\section{5-Bromo-20-deoxyuridine proliferation assay}

For cell proliferation analysis, microglial cells were seeded onto 96-well plates and transfected with the siRNA/dendrimer complexes. After $48 \mathrm{~h}$, the cells were stimulated with GCM for the next $24 \mathrm{~h}$. 5-Bromo20-deoxyuridine (BrdU; $10 \mu \mathrm{M}$ ) was added for the last $20 \mathrm{~h}$ of treatment. Subsequently, cells were fixed and BrdU incorporation was determined using a Cell Proliferation ELISA BrdU kit (Roche, Mannheim, Germany), according to the manufacturer's protocol.

\section{Statistical analysis}

Experiments were carried out on 3-5 independent primary microglial cultures. Statistical analyses were performed using Student's t-test or, for multiple comparisons, with two-way ANOVA followed by post hoc Tukey test using the GraphPad Prism software (GraphPad, Inc., CA, USA). The p-values $<0.05$ were considered to be statistically significant, and significance is marked on the graphs as ${ }^{*} \mathrm{p}<0.05,{ }^{* *} \mathrm{p}<0.01$ and ${ }^{* * *} \mathrm{p}<0.001$. Nonsignificant differences are marked as ns.

\section{Results}

Formation of small \& robust siRNA/dendrimer complexes to protect siRNA from degradation In line with previous results from our group [36], the dendrimer $\mathrm{AD}$, which is positively charged at physiological $\mathrm{pH}$, was able to complex the negatively charged siRNA via electrostatic interactions. As shown by the gel mobility shift assay (Figure 2A), AD greatly retarded the migration of siRNA, when the N/P ratio (the number of dendrimer amine terminals versus the number of siRNA phosphates) was above 2.5. The formed siRNA/AD complexes were small and uniformly spherical in shape, as revealed by TEM (Figure 2B). Also, dynamic light scattering (DLS) analysis confirmed that the siRNA/AD nanoparticles were small in size ( $37 \mathrm{~nm})$ (Figure $2 \mathrm{C})$, and characterized by 
(A)

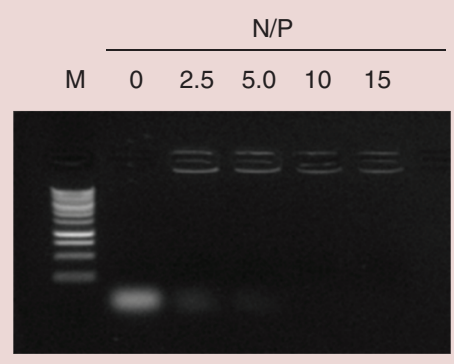

(C)

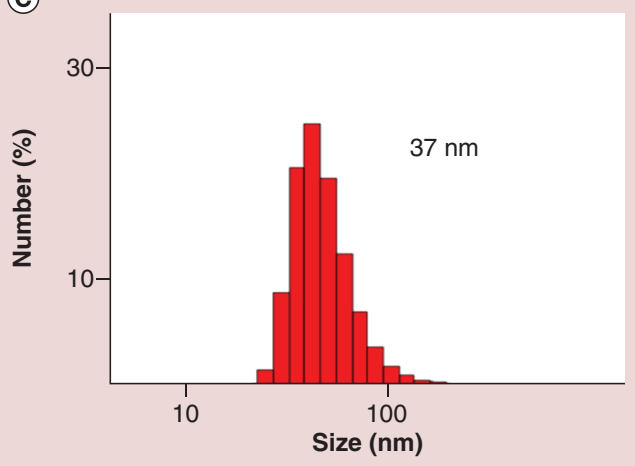

(E)

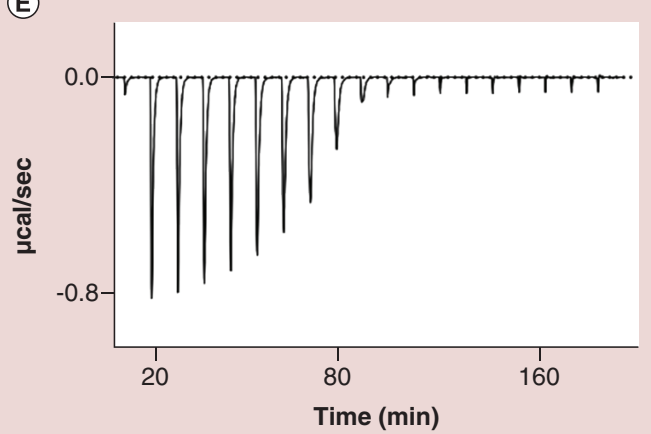

(B)

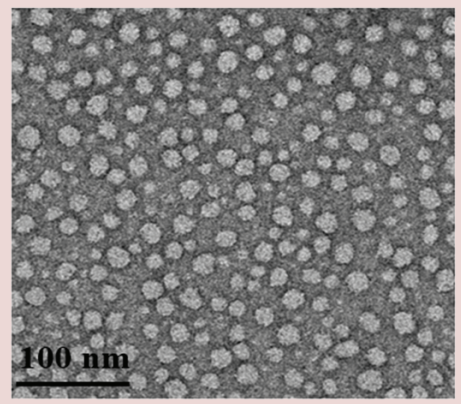

(D)
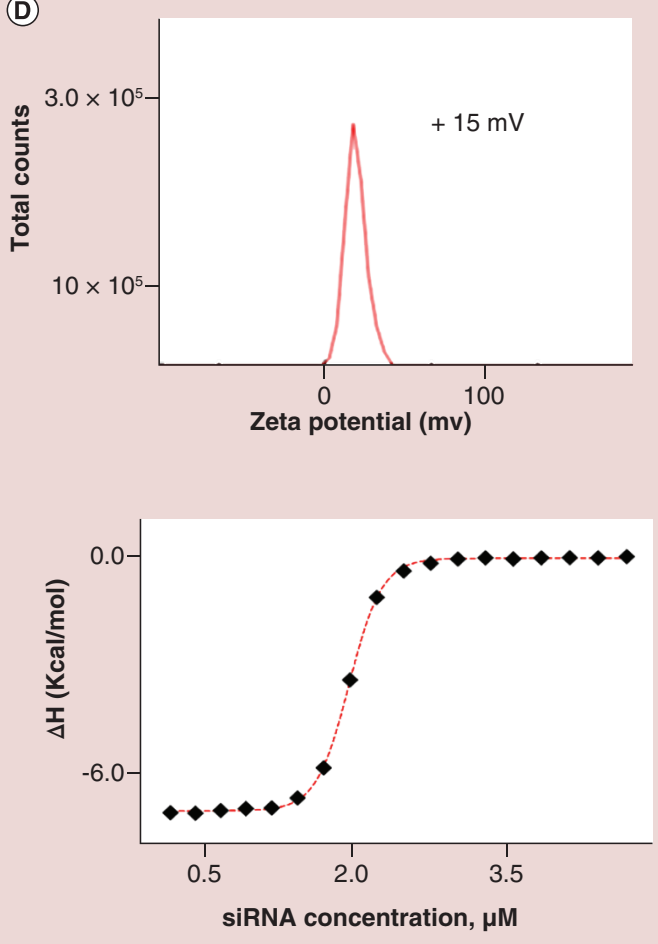

(F)

Time (min)

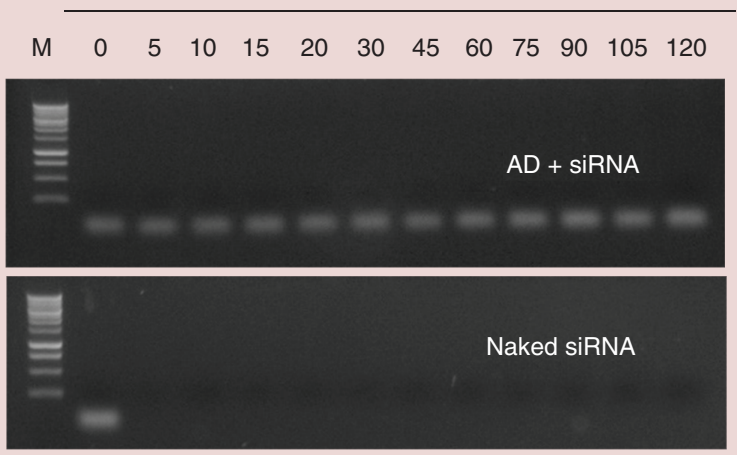

Figure 2. The amphiphilic dendrimer vector $A D$ forms small and robust nanoparticles with siRNA, and protects the siRNA from degradation. (A) AD binding with siRNA (80 $\mathrm{ng}$ ) in diethylpyrocarbonate-treated water at different N/P ratios varying from 2.5 to 15 was assessed using agarose gel electrophoresis. Naked siRNA was used as the control $(\mathrm{N} / \mathrm{P}$ ratio $=0)$. (B) transmission electron microscopy imaging, $(C)$ Dynamic light scattering and $(\mathrm{D})$ zeta potential analysis of the siRNA/AD complexes at an N/P ratio of 10. (E) Isothermal titration calorimetry raw (left panel) and integrated (right panel) data for titration of $A D$ with siRNA in ultrapure water $\left(T=25^{\circ} \mathrm{C}\right)$. The dotted red line represents data fitting with a sigmoidal function. All experiments were run in triplicate. (F) The siRNA with (top) and without (bottom) AD was incubated with $0.25 \mu \mathrm{g} / \mu \mathrm{l}$ RNase $A$ at room temperature for $30 \mathrm{~min}$, and then with $1.0 \%$ SDS at $4^{\circ} \mathrm{C}$. The siRNA was analyzed by agarose gel electrophoresis. 
a narrow polydispersity (0.20). In addition, the zeta potential of the siRNA/AD nanoparticles was around $+15 \mathrm{mV}$ (Figure 2D), implying stable colloidal property. Thermodynamics assessments using ITC showed a substantial exothermic (i.e., favorable), enthalpic contribution of the interactions between the siRNA molecules and the dendrimer $\mathrm{AD}(\Delta \mathrm{H}=-6.53 \mathrm{kcal} / \mathrm{mol})$, reflecting the essentially electrostatic nature of the driving force leading to the formation of stable siRNA/AD complexes (Figure 2E). The related entropy variation upon siRNA/AD binding was also favorable (i.e., positive $\mathrm{T} \Delta \mathrm{S}=3.25 \mathrm{kcal} / \mathrm{mol}$ ), and could be ascribed to the release of water and counter ions from the siRNA/AD binding interface into bulk solvent. Overall, the free energy of binding $\Delta \mathrm{G}$ was largely negative $(-9.78 \mathrm{kcal} / \mathrm{mol})$, supporting the thermodynamically spontaneous and largely favorable formation of siRNA/AD complexes. Indeed, the siRNA/AD complexes were very stable and effectively protected siRNA from enzymatic digestion (Figure 2F, top); whereas, free siRNA was degraded rapidly (5 min) upon treatment with RNase A (Figure 2F, bottom). Altogether, our data demonstrate that the AD can form small and stable nanoparticles with siRNA, which effectively protect the siRNA from degradation.

\section{Effective cellular uptake \& intracellular trafficking of siRNA in microglia}

Cell uptake is one of the critical steps to ensure successful siRNA delivery. We therefore verified the uptake of the siRNA/AD complexes in microglial cells using a reference siRNA labeled with a red fluorescent tag (denoted as siGlo hereafter). As shown in Figure 3, uptake and accumulation of the siGlo/AD complexes was evident in the majority of the primary microglial cells derived from both rats (Figure 3A) and mice (Figure 3B), with 80-90\% cells showing fluorescence. In contrast, microglia treated with naked siGlo had almost no notable fluorescent signal (Supplementary Figure 1). This highlights the high efficiency of siRNA delivery mediated by the dendrimer AD. Also, treatment with either the $\mathrm{AD}$ dendrimer alone or the siGlo/AD complexes did not affect cell morphology, and the microglia remained elongated and bipolar (Figure 3A \& B). These observations constituted a first indication that the dendrimer $\mathrm{AD}$ is able to mediate safe siRNA delivery to primary microglia.

Cell internalization of nanosized particles is often associated with the endocytotic uptake pathway, in which the nanoparticles are first entrapped in the endosomes and then released into the cytosol via endosomal escape. Accordingly, we analyzed the intracellular trafficking of the red fluorescence-labeled control siRNA (siCtrl-Glo)/dendrimer complexes in mouse microglia after immunostaining of cells with a green fluorescence-labeled antibody against the early endosomal marker EEA1. After $1 \mathrm{~h}$ incubation of the microglial cells with the siCtrl-Glo/dendrimer complexes, co-localization of the fluorescent signals from siCtrl-Glo (red) and endosomal EEA1 (green) was observed as yellow signals under the confocal microscope (Figure 3C). This indicated that the siRNA/dendrimer complexes were entrapped in the endosomes. Importantly, after 3-h incubation, dispersed and smeared red fluorescent signals were observed in the cytosol (Figure 3D). These results indicate that the siRNA/dendrimer complexes had indeed escaped from the endosomes into the cytosol. Collectively, these results highlight that the dendrimer AD nanovector mediated safe and efficient cellular uptake and intracellular trafficking of siRNA in microglia. This prompted us to perform further functional studies of siRNA-mediated gene silencing.

\section{The siRNA delivery mediated by AD without inflammatory or pro-invasive microglial response}

Before performing functional studies using siRNA-mediated gene silencing in microglia, it is of great importance to verify that the delivery vectors do not nonspecifically affect basal or induced gene expression. We therefore examined the expression of a panel of genes, characteristic for either inflammatory (iRf7, iNos, COX2) or protumoral/proinvasive (YKL40, Arg1, Id1, MMP14, cMyc, CX3CR1) microglial responses [46]. Microglial cells were untreated, or treated with $\mathrm{AD}$ alone, or treated with the siRNA/AD complexes using a nontargeting siRNA (siCtrl). Neither $\mathrm{AD}$ alone nor the siCtrl/AD complexes significantly changed the expression of any of the tested genes in rat microglia cultures as compared with untreated control cultures (Figure 4A). In mouse microglial cells, due to the limited number of cells in primary murine glial cultures, only a subset of these genes was tested (Figure 4B). All the selected genes are major markers for distinct microglial phenotypes: IRF7 and INOS for the inflammatory phenotype, and ArgI and $c M y c$ for the pro-invasive phenotype. Neither the dendrimer AD nor the siCtrl/AD complexes considerably influenced the expression of all these selected genes (Figure 4B). In addition, neither AD alone nor the siCtrl/AD complexes upregulated the protein levels of inflammation mediators such as iNos and Cox2, as shown in Figure 4C. It is to mention that primary microglia cultures treated with glioma conditioned media (GCM) and a bacterial lipopolysaccharide (LPS, $100 \mathrm{ng} / \mathrm{l}$ ) were used as the negative and positive controls for inflammatory activation, respectively. 
(A)
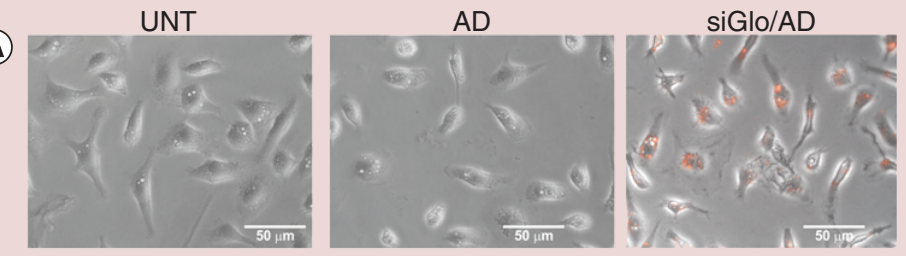

(B)
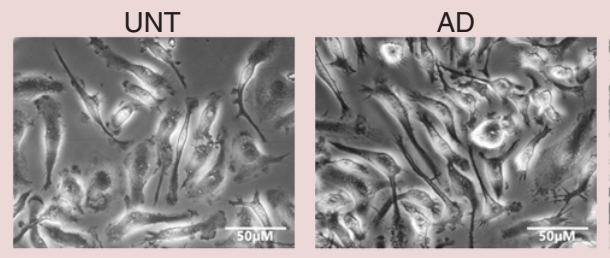

siGlo/AD

(C)
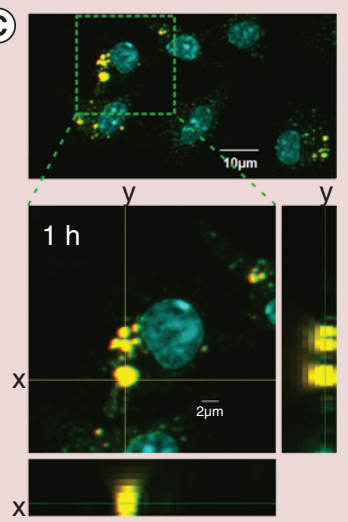

(D)
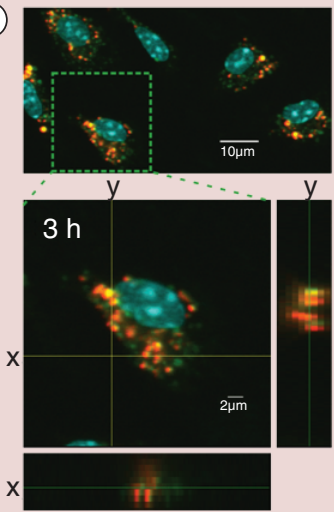

Figure 3. The amphiphilic dendrimer vector AD facilitates efficient delivery of siRNA to microglia. The siRNA transfection indicator labeled with a red fluorescent tag (siGlo) was used. Cultures of rat microglial cells and mouse microglial cells were UNT or incubated with the dendrimer AD alone or with the siGlo/AD complexes (12.5 nM siRNA with $A D$ at N/P 10). Representative images of rat (A) and mouse (B) microglial cells were taken $24 \mathrm{~h}$ post-transfection and show unchanged cell morphology and high transfection efficiency. Confocal images of mouse microglia incubated for 1 (C) or $3 \mathrm{~h}$ (D) with red fluorescent control siRNA (siCtrl-Glo)/AD complexes and stained with the green fluorescent anti-EEA1 antibody to visualize endosomes. In cells transfected with the siCtrl-Glo/AD complexes, $z$-stack projections and orthogonal views along the $x$ and $y$ axes demonstrated the presence of red-labeled siCtrl-Glo in the endosomes at $1 \mathrm{~h}$ and endosomal escape at $3 \mathrm{~h}$ post-transfection.

AD: Amphiphilic dendrimer; UNT: Untreated.

We next compared the performance of the AD dendrimer in siRNA delivery with that of commercial viral and nonviral vectors. Primary rat microglia cultures were thus treated with: a lentiviral vector carrying a nontargeting shRNA (shLuc) and a fluorescent labeled nontargeting siRNA (siGlo) complexed with the transfection reagent Viromer Blue (named as Viromer hereafter). Both Viromer and lentiviral vectors delivered their cargos very efficiently (Supplementary Figure 2A \& B); however, the IRF7 mRNA was found to be strongly upregulated, by almost 100-fold and 250-fold at 1 day post-treatment, in shLuc/LV- and siCtrl/Viromer-transfected microglial cells, respectively (Figure 4D). At the same siRNA concentration $(25 \mathrm{nM})$ as used in the transfection with Viromer, siRNA/AD complexes induced less than a twofold increase in the level of IRF7 mRNA at 1 day postexposure and the observed increase was not statistically significant (Figure 4D). The IRF7 gene encodes interferon response factor 7, a transcription factor that is activated as a part of the inflammatory response and is a key regulator of the type I (IFN $\alpha$ and IFN $\beta$ ) interferon response during immune responses [47]. In our studies on microglia, robust upregulation of IRF7 mRNA by several dozen fold was the main indicator that inflammation was induced. It is worth noting that even transient stimulation is able to deregulate gene expression and may alter microglia behavior [48]. In addition, the induction of the inflammatory response in siCtrl/Viromer-transfected microglial cells was further corroborated by the observed upregulation of genes associated with antiviral responses such as 
(A)

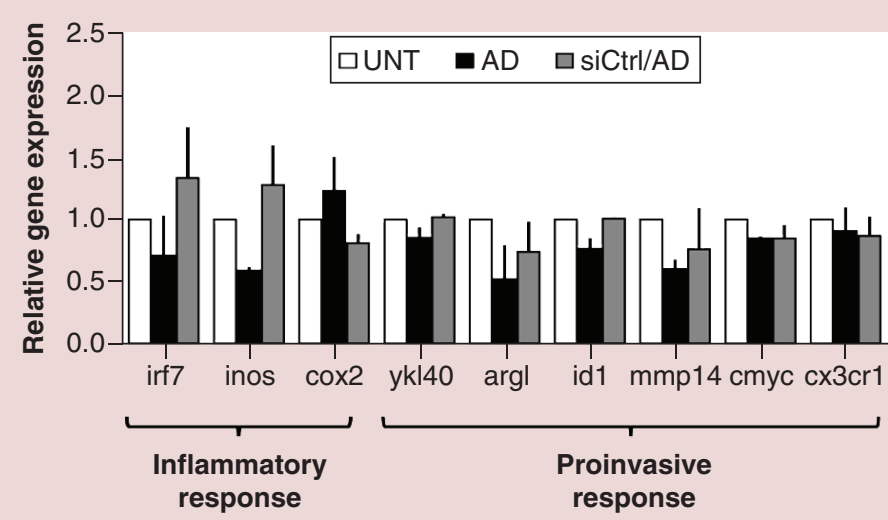

(C)

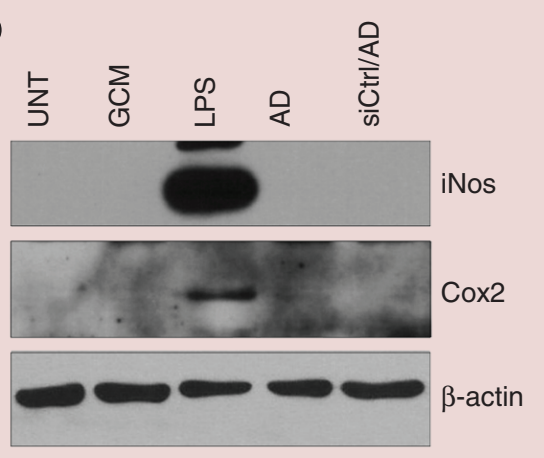

(B)

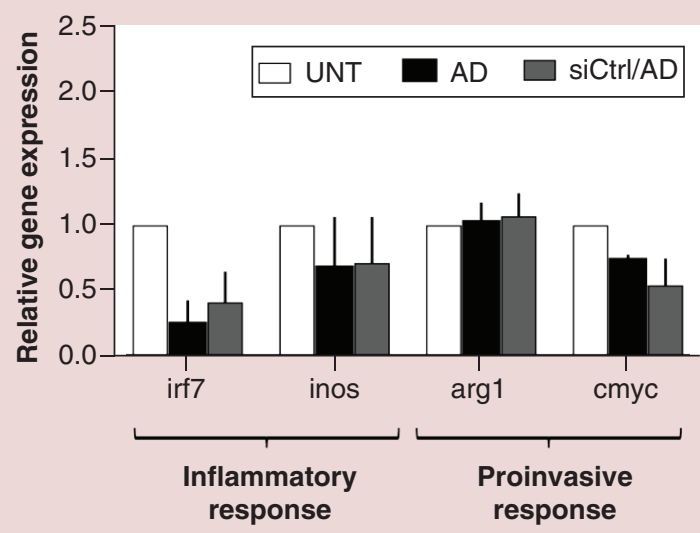

(D)

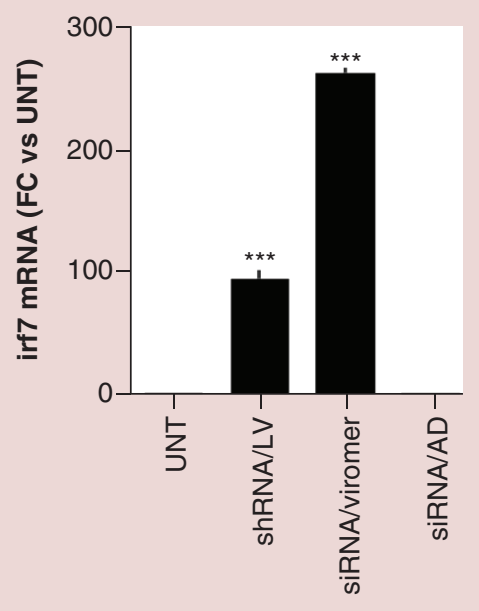

Figure 4. The siRNA delivery mediated by amphiphilic dendrimer does not induce inflammatory or pro-invasive response of microglia. (A, C) AD-mediated siRNA delivery to rat and (B) mouse microglia does not upregulate inflammatory or pro-invasive response genes (A-D), while both a lentiviral vector and a commercial nonviral vector Viromer induce strong inflammation (D). Cultures of rat microglial cells and mouse microglial cells were UNT or incubated with the dendrimer AD alone or with the siRNA/AD complexes (12.5 nM siRNA with $A D$ at N/P 10). For gene expression assays, the siCtrl was employed. Gene expression in (A) rat and (B) mouse microglial cells was measured using quantitative real-time PCR $48 \mathrm{~h}$ post-transfection ( $\mathrm{N}=3$, average \pm standard deviation). (C) The protein levels of the inflammation mediators iNos and Cox 2 were analyzed in rat microglial cultures by western blotting at $72 \mathrm{~h}$ post-transfection. Membranes were stripped after probing with antibodies and detection of $\beta$-actin ensured equal protein loading. Microglia cultures treated with glioma conditioned medium and $100 \mathrm{ng} / \mathrm{ml}$ bacterial lipopolysaccharide were used as negative and positive controls for the inflammatory responses, respectively. (D) Primary rat microglia cultures were transfected using lentiviral vector carrying a control shRNA targeting the luciferase gene (shLuc/LV), the nonviral vector Viromer complexed with $25 \mathrm{nM}$ of the siCtrl and the dendrimer vector AD complexed with $25 \mathrm{nM}$ of the control siCtrl (at N/P 10). The mRNA level of irf7 in primary rat microglia cultures transduced with the shLuc/LV, transfected with the siCtrl/Viromer and siCtrl/AD at 1 day after transfection is presented as fold change relative to untreated cells.

AD: Amphiphilic dendrimer; siCtrl: Nontargeting siRNA control; UNT: Untreated.

$I F N \beta$, IRF7 and ZBP1 (Supplementary Figure 3). Taken together, these results show that, in contrast to the tested viral and nonviral carriers, the AD did not induce inflammatory gene expression, while maintaining a high siRNA delivery efficiency.

With the goal of maximizing the delivery efficiency while maintaining the minimal side-effects of the dendrimer vector $\mathrm{AD}$, we searched for an optimal siRNA concentration using either the fluorescently tagged siGlo as the transfection indicator (Figure $5 \mathrm{~A} \mathrm{\&} \mathrm{B}$ ) or the nontargeting siCtrl for the gene expression analysis (Figure 5C \& D). We found that the siGlo/AD complexes with siRNA concentrations of 12.5 and $25.0 \mathrm{nM}$ had very high cell 

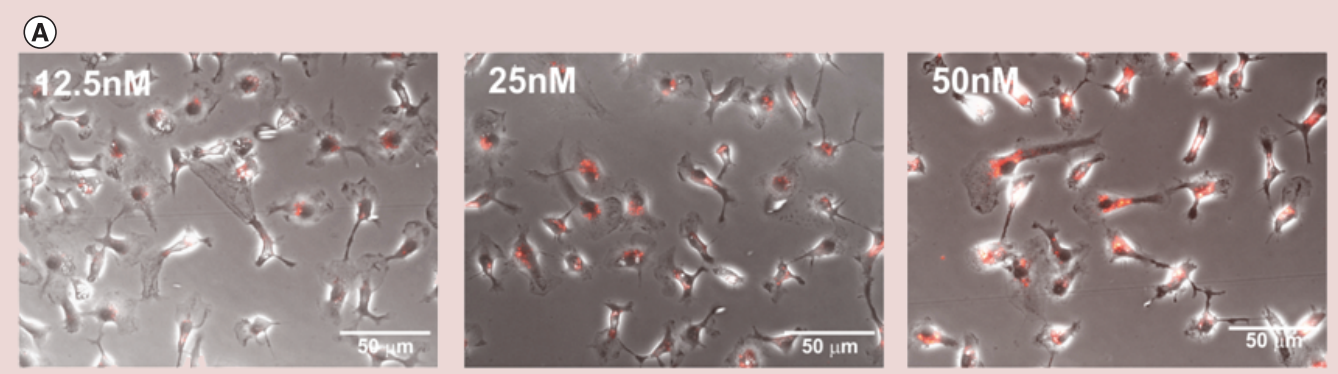

(B)
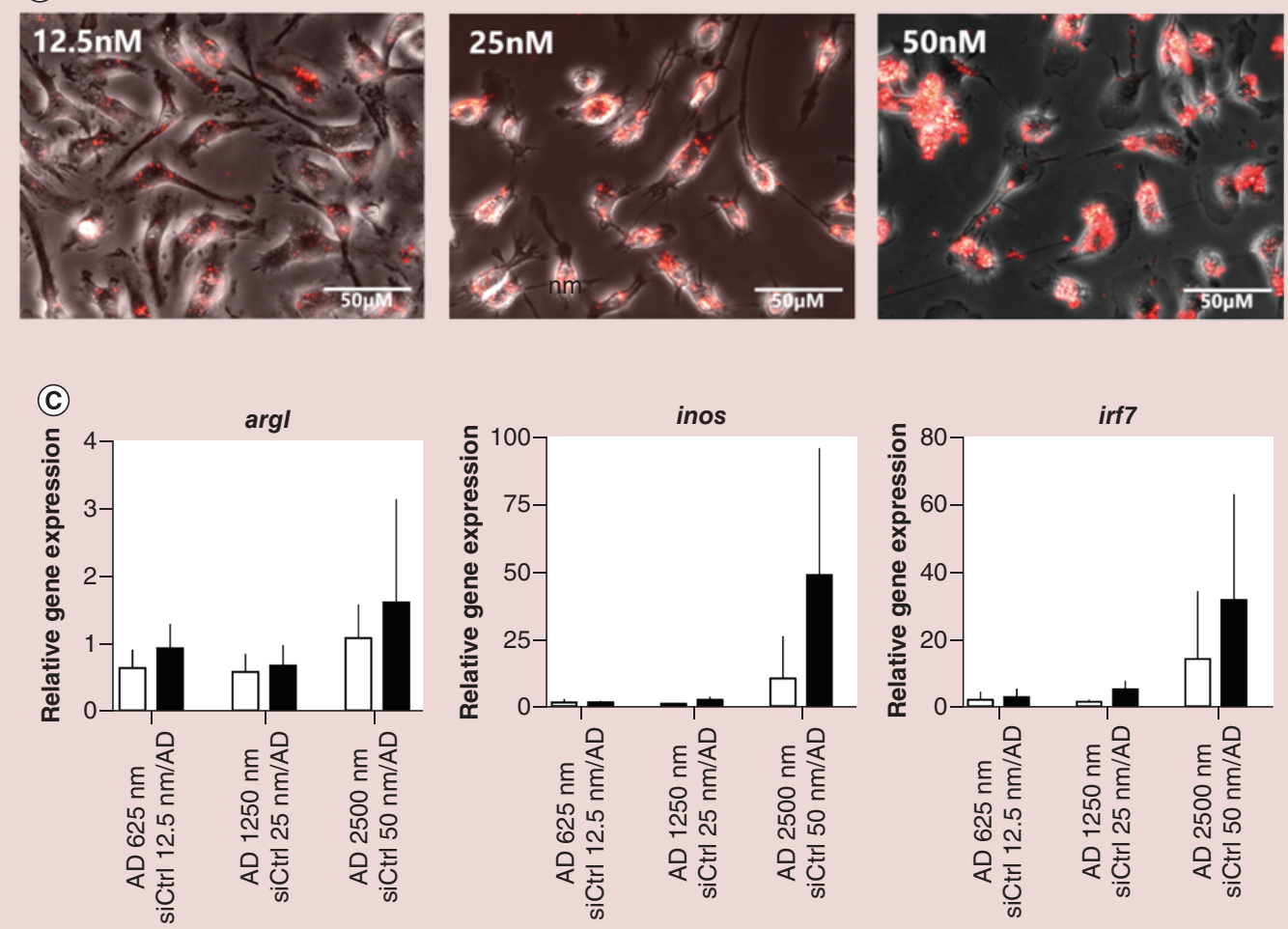

(D)
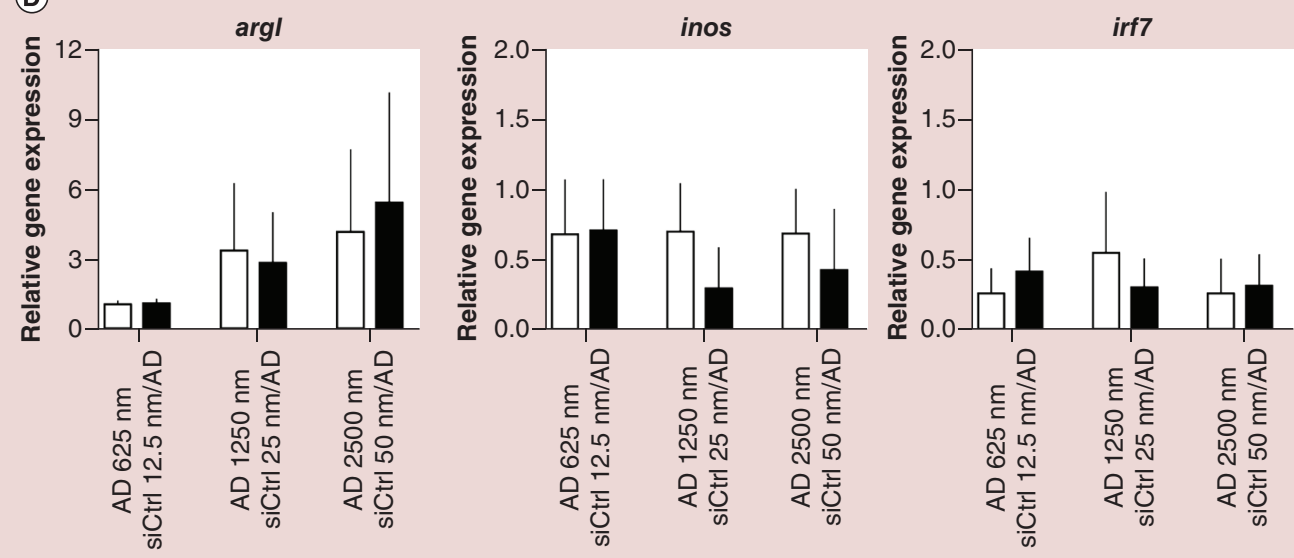

Figure 5. Optimization for the safe siRNA delivery to microglia using the dendrimer vector AD. (A \& B) AD-mediated delivery of siRNA at increasing concentrations to microglia, and the effects on cell morphology and (C \& D) gene expression in microglia. The siGlo siRNA was used to study uptake efficacy by microscopy analysis and the siCtrl was used to study the effects on gene expression. Representative images of (A) rat and (B) mouse microglia were taken $24 \mathrm{~h}$ post-treatment with the siGlo/AD complexes at different concentrations of siRNA at a constant ratio N/P $=10$. Gene expression in (C) rat and (D) mouse microglia was measured using quantitative real-time PCR at $48 \mathrm{~h}$ after treatment with $A D$ alone (white bars) or with the siCtrl/AD complexes (black bars). The siRNA and AD concentrations are indicated. Bars represent the mean ( $n=3, \pm$ standard deviation) gene expression relative to untreated cells. AD: Amphiphilic dendrimer; siCtrl: Nontargeting siRNA. 
uptake and siRNA delivery, without inducing notable morphological alterations in rat microglial cells (Figure 5A) or mouse microglial cells (Figure 5B). Concomitantly, no significant alteration in gene expression was observed in rat microglial cells (Figure 5C) or mouse microglial cells (Figure 5D) when treated with the siCtrl/AD complexes with siRNA concentrations of 12.5 and $25.0 \mathrm{nM}$. At the highest siRNA concentration tested $(50 \mathrm{nM})$, a slight activation of microglia was observed, as evidenced by the moderate shift towards the amoeboid cell shape of mouse microglia (Figure 5B) together with the increases of $A R G 1$, iNOS and IRF7 expression (Figure 5C \& Figure 5D), which were more pronounced in rat microglia (Figure $5 \mathrm{C}$ ). As a highly variable inflammatory response is an inherent feature of primary cultures, some increase in gene expression was observed in a few experiments when the highest siRNA concentration was used. However, these gene expression changes were not statistically significant. In order to minimize the side effects and achieve best delivery performance, we used $12.5 \mathrm{nM}$ siRNA with the dendrimer $\mathrm{AD}$ at a N/P ratio of 10 for further studies.

Functional siRNA delivery in a cellular model of microglia-glioma interaction

With the optimized delivery conditions at hand, and with the goal of specifically interfering with glioma-related responses, we next tested the dendrimer $\mathrm{AD}$ for functional siRNA delivery to block selected gene expression in microglia. It is to mention that the molecular events underlying glioma-induced polarization of microglia can be mimicked in vitro by applying GCM to primary cultures of microglial cells [39,49], which leads to the activation of specific signaling pathways, increased proliferation and global changes in gene expression, including upregulation of genes such as $A R G 1, c M y c, I D 1, I D 3$ [46]. Indeed, as shown in Figure 6, GCM significantly enhanced proliferation of rat microglial cells (Figure 6A) and promoted the expression of the protumorigenic and pro-invasive genes ARG 1 , cMyc, ID1, ID3, SMAD7, MARK1, CX3CR1 and GPR34 (Figure 6B). Treatment with AD alone or the siCtrl/AD complexes did not affect the basal and GCM-stimulated proliferation of microglia (Figure 6A), neither influenced the expression of the GCM-induced genes (Figure 6B). Cells treated with either AD or the siCtrl/AD complexes retained their ability to proliferate and respond to GCM. These functions of microglia, as judged by the evaluation of expression of selected genes, were preserved even when double the concentration of siRNA/AD complexes was added to the culture medium (Supplementary Figure 4).

One of the genes upregulated by GCM in microglia is ID1 [46]. Id1 is a transcription regulator, which controls cell growth and differentiation by preventing basic helix-loop-helix transcription factors from binding to DNA [50]. Id factors bind to cell cycle regulators such as $\mathrm{Rb}$ proteins and Ets transcription factors that play key roles in cell cycle regulation and thus cell proliferation [51]. Id1 is a negative regulator of myeloid cell differentiation during hematopoiesis [52] and also during tumor progression [53]. Previous work has revealed that ID1, ID3 and $c M y c$ mRNAs are upregulated in GCM-stimulated microglia, and the increase in ID1 expression precedes the changes in ID3 and $c M y c$ expression [46]. Accordingly, in the present study, the levels of ID1, ID3 and $c M y c$ mRNA were increased after adding GCM (Figure 6B). We then studied the outcomes of the AD-mediated delivery of an IDIspecific siRNA (referred to as siId1 hereafter) on the reduction of the level of IDI mRNA, and the consequent effects on GCM-induced upregulation of the other genes. Indeed, transfection of rat microglial cells with the siId1/AD complexes significantly decreased the level of the $I D 1 \mathrm{mRNA}$ (Figure 7A). The level of ID1 protein was also reduced (Figure 7B). Moreover, the expression of $c M y c$ and $I D 3$ was downregulated at both mRNA (Figure 7C \& D) and protein (Figure 7B) levels in microglial cells transfected with the siId1/AD complexes. The level of phosphorylated $\mathrm{Rb}$ (phRb) also dropped following IDI knockdown (Figure 7B), which is consistent with the postulated mode of the ID1 action [51].

In order to demonstrate the functional effect of IDI knockdown on microglial cells, we next performed global gene expression analysis. RNA sequencing was used to measure the levels of mRNA expression and to generate transcriptomic profiles in GCM-stimulated microglia treated with AD-delivered IdI-targeting siRNA and control siRNA siCtrl. Using computational analysis, we identified genes that were differentially expressed between the two conditions and classified these genes into functional categories defined in the KEGG pathways database. The results of pathway enrichment analysis are shown in Supplemenatry Figure 5. Notably, we found significant downregulation of genes related to the cell-cycle and DNA replication after IDI knockdown (Figure 7E), which indicates the decreased proliferative activity of the microglia. The downregulated genes include those coding for proliferation markers, such as proliferating cell nuclear antigen (PCNA); the E2F2 and E2F5 transcription factors, which play a crucial role in the control of cell cycle and subunits of the DNA polymerases delta and epsilon, which are involved in DNA replication (Figure 7F). 


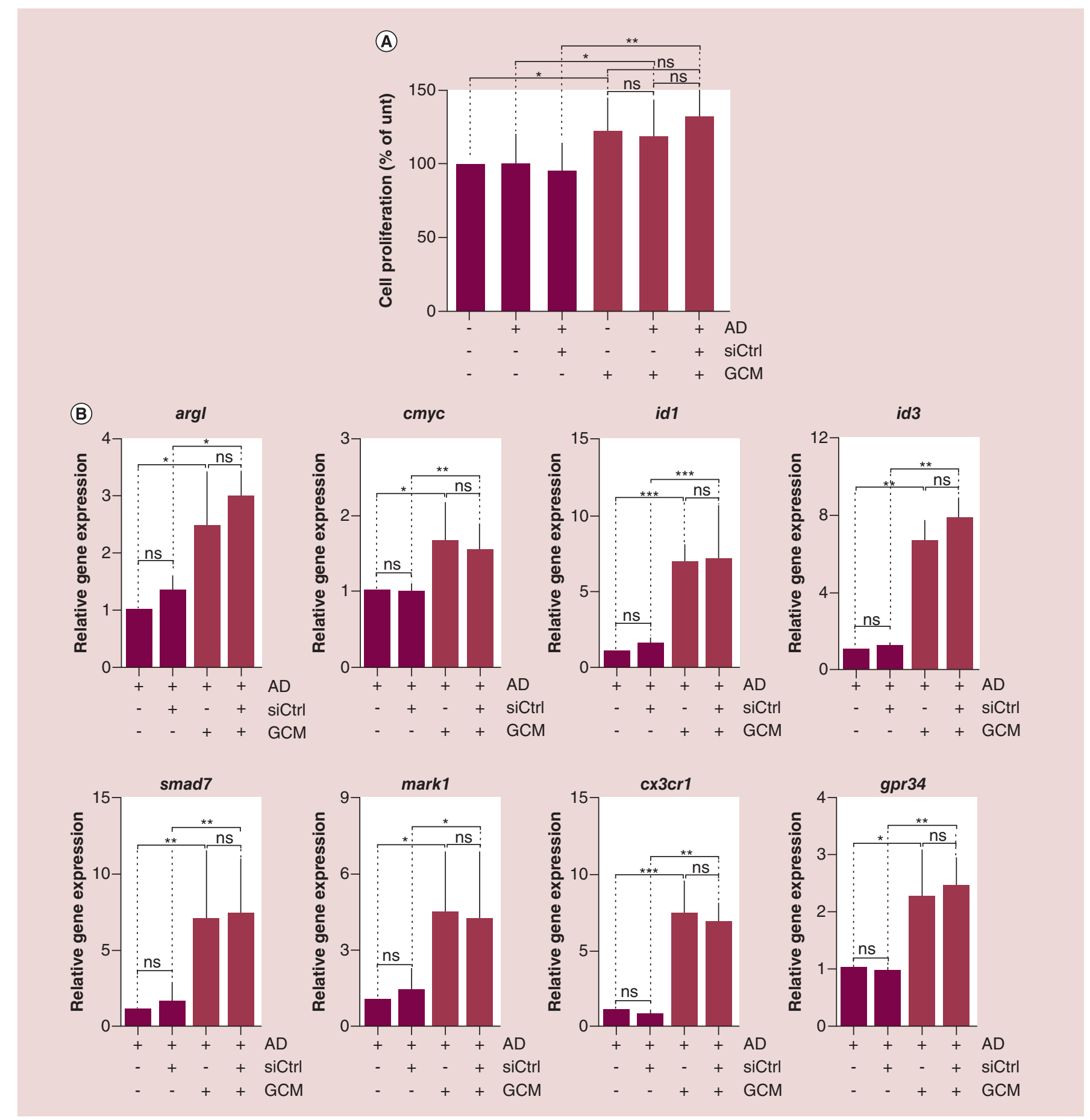

Figure 6. Amphiphilic dendrimer alone or its complex with the control siRNA does not affect glioma conditioned medium-induced responses of microglia. Control or GCM-treated rat microglial cells were incubated with AD or siCtrl/AD complexes (12.5 nM siRNA with $A D$ at $N / P=10)$ as indicated. Proliferation of microglia was evaluated using BrdU incorporation assay at $72 \mathrm{~h}$ post-treatment $(A)$.

Microglial responses to GCM were determined by qPCR gene expression analyses at $48 \mathrm{~h}$ post-treatment (B). Gene expression levels in siCtrl/AD-transfected microglia are shown relative to $A D$ alone $(n=3$, mean \pm standard deviation). Groups were compared using two-way ANOVA with a post hoc Tukey test for multiple comparisons: ${ }^{*} p<0.05 ;{ }^{* *} p<0.01 ; * * * p<0.001$.

$A D$ : Amphiphilic dendrimer; BrdU: 5-Bromo-20-deoxyuridine; GCM: Glioma conditioned medium; ns: Not significant; qPCR:quantitative real-time PCR; siCtrl: Control siRNA. 

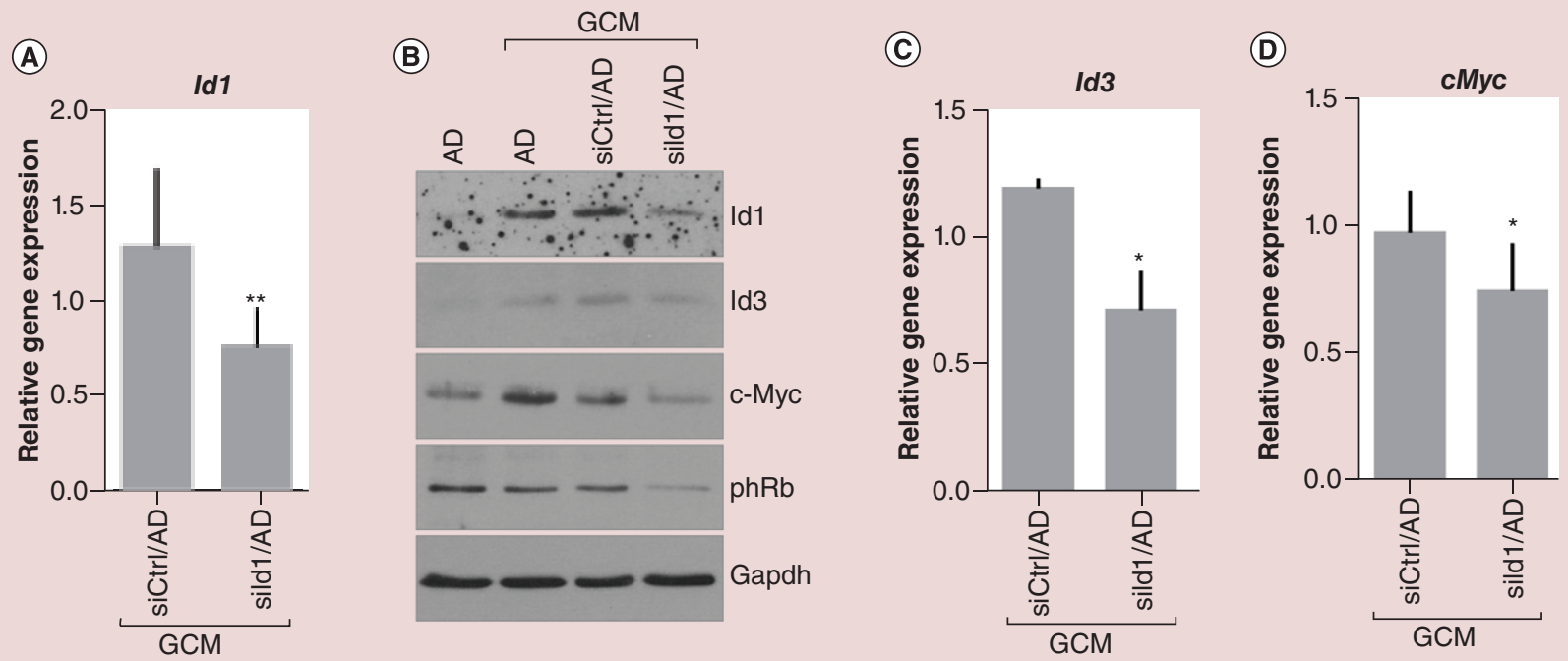

(E)

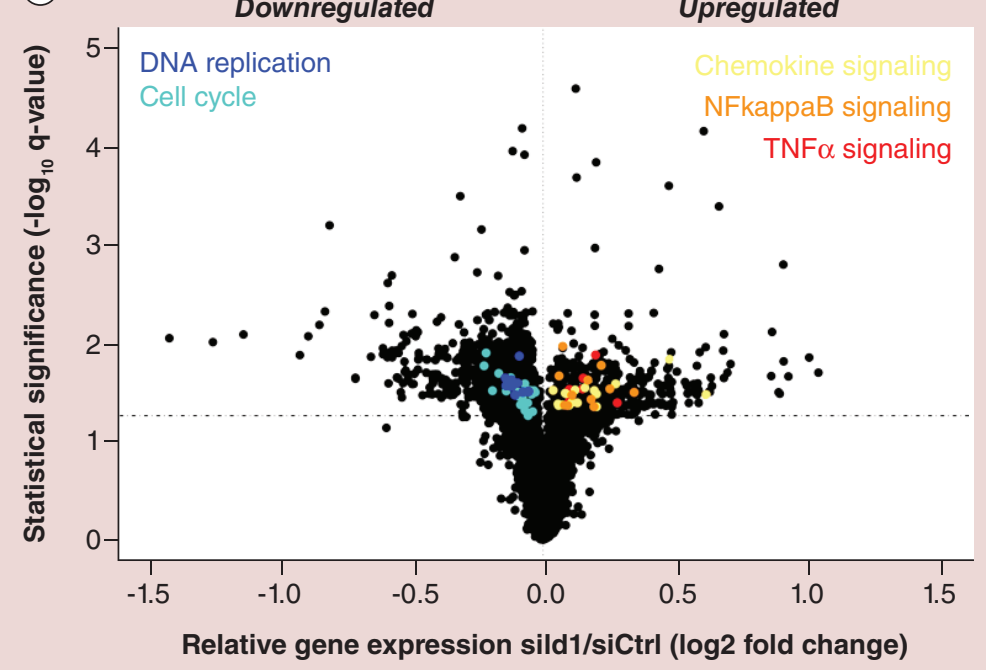

(F)

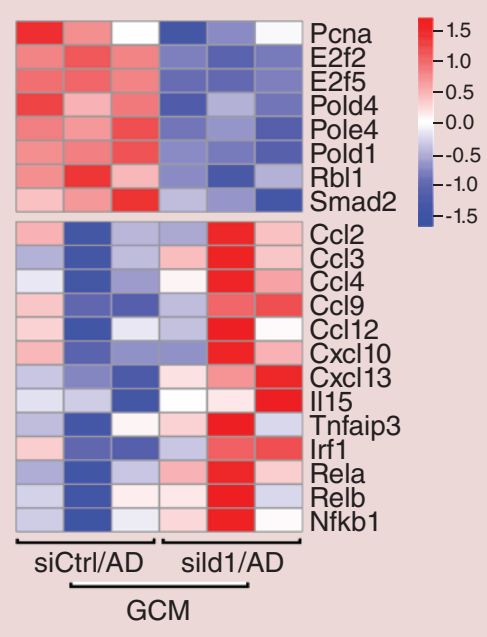

Figure 7. Specific siRNA/amphiphilic dendrimer complexes effectively silence the glioma conditioned medium-induced expression of ID1 and the related downstream genes in primary rat microglia. Rat microglia were transfected with $A D$ alone, siCtrl/AD or sild1/AD. Both siRNAs were complexed with $A D$ at N/P = 10, $12.5 \mathrm{nM}$ siRNA. (A, C \& D) The levels of mRNA and (B) protein were analyzed $72 \mathrm{~h}$ post-transfection using qPCR and western blotting, respectively. Microglia were stimulated with GCM for the last $6 \mathrm{~h}$ of treatment. Gene expression is presented as fold change relative to the treatment with $A D$ alone and stimulation with $G C M(n=3$, mean \pm standard deviation). Statistically significant differences between cultures treated with sild1/AD + GCM and siCtrl/AD + GCM were evaluated using paired Student's t-test. * $\mathrm{p}<0.05 ;{ }^{*} \mathrm{p}<0.01$. The effects of $I d 1$ knockdown on the protein levels of Id1, Id3, cMyc and phRb were evaluated using western blotting. Detection of Gapdh confirmed equal protein loading. (E) The Volcano plot shows down- and upregulated genes (log2 fold change $<0$ and log2 fold change $>0$, respectively) after Id 1 silencing in GCM-stimulated rat microglia relative to siCtrl/AD-treated, GCM-stimulated cultures. Each dot above the dashed line (corresponding to $q$-value $=0.05$ ) represents one significantly changed gene. The genes from selected functional KEGG categories are marked in different colors, as indicated. (F) Z-score heat maps for selected genes represent the relative change in gene expression in microglia treated with sild1/AD as compared with siCtrl/AD, after GCM stimulation in both cases. Three replicates per treatment.

AD: Amphiphilic dendrimer; GCM: Glioma conditioned medium; phRB: Phosphorylated Rb; qPCR: quantitative real-time PCR; siCtrl/AD: Complex of $A D$ with control siRNA; sild1/AD: Complex of AD with the ID1-targeting siRNA. 
Moreover, silencing of ID1 in GCM-stimulated microglia led to upregulation of genes related to inflammation, immune response and chemotaxis, which fall into chemokine, TNF $\alpha$ and NF- $\mathrm{KB}$ signaling pathways according to KEGG (Figure 7E). This suggests restoration of the ability to initiate an immune response, which is largely suppressed in microglia exposed to glioma-secreted factors [46,54]. In particular, the upregulated genes code for several cytokines and chemokines (such as Ccl2, Ccl3, Ccl4, Ccl9, Ccl12, Cxcl10, Cxcl13 and Il15), mediators of the response to TNF $\alpha$ (including Tnfaip3 and Irf1), and major subunits of the NF- $\mathrm{BB}$ transcription factor (Rela, Relb and NFkb1), which are involved in cytokine-mediated immune and inflammatory responses (Figure 7F).

Collectively, these results show that the IDI siRNA delivered by the dendrimer AD nanovector effectively inhibited IDI gene expression in primary microglial cultures, leading to biological effects on genes controlling the cell cycle and immune response.

\section{Discussion}

In this study, we demonstrated that the dendrimer AD safely and successfully delivered siRNA into rat and mouse microglial cells in primary culture, leading to downregulation at both mRNA and protein levels, with subsequent biological effects. In addition, $\mathrm{AD}$ was harmless to microglia and did not affect their basal function or immune gene expression. This AD was designed for effective siRNA delivery by ensuring the formation of strong and small complexes with siRNA, which protect siRNA from degradation, mask its negative charge and facilitate its cellular uptake. Importantly, this dendrimer AD allowed for effective siRNA delivery at a low siRNA concentration, and had no effect either on the morphology of primary microglia derived from murine and rat brains or on the expression of inflammatory and pro-invasive response genes in these cells.

It should be mentioned that the two commonly used methods for genetic manipulation using RNAi, in other words, treatment with lentiviral vector carrying shRNA and delivery of siRNA with the nonviral vector Viromer, both led to a strong induction of IRF7 mRNA in microglia cultures (Figure 4D). IRF7 encodes a key regulatory transcription factor of the type I IFN response, a hallmark of inflammation [47]. Also, another frequently used transfection reagent, lipofectamine, was recently reported to induce type I IFN inflammatory signaling in both RAW 264.7 macrophage-like cells and primary bone marrow-derived macrophages [55]. Since immune dysregulation can persist long after the initial insult, functional studies on microglia treated with previously available vectors were largely hindered [48]. In view of the results reported here, the AD constitutes the long-searched and superior vector for safe and effective siRNA delivery for the purpose of functional studies on primary microglial cells, when compared with the commercially available viral and nonviral vectors, which induce strong inflammatory effects in primary microglia cultures.

As acute or chronic alterations in microglia are often associated with numerous brain pathologies, we further explored microglia responses in a cellular model of glioma-induced microglia activation, which mimics the interactions between brain tumor cells and microglia within the tumor microenvironment. Microglia, when 'educated' by the tumor, acquire a specific phenotype devoid of inflammatory components $[13,46]$. The GCM-induced responses of microglia were not affected in the presence of either $\mathrm{AD}$ or its complexes with siRNA, even at twofold higher concentrations as eventually used in functional assays. This emphasizes again the low toxicity of the AD as an siRNA carrier for primary microglial cells. The lack of a priming pro-inflammatory effect provides more reliable data for evaluation of the target gene knockdown.

The transcription regulator $I d I$, which controls proliferation/differentiation in many cells, has been hypothesized to act as a master switch in microglia activation by glioma-derived factors. The AD-mediated delivery of a specific anti-IdI siRNA triggered downregulation of the target $I d I \mathrm{mRNA}$ and its protein product in microglia, which allowed exploration for the first time of the functions of $I d 1$ in GCM-stimulated microglia. Transcriptome analysis showed that silencing of $I d I$ in microglia affected the expression of cell cycle/proliferation-related genes, while also uncovering a program regulating inflammatory gene expression, which was suppressed by glioma-derived factors. This corroborates the recently postulated role of $I d I$ in tumor-induced immunosuppression via inhibition of myeloid cell maturation [53]. Collectively, our results demonstrate the excellent performance of AD-mediated delivery of siRNA and highlight the potential of the $\mathrm{AD}$ as a potent and promising nanovector for efficient and nonnoxious siRNA delivery to primary microglial cells.

\section{Conclusion}

We report in this work an amphiphilic PAMAM dendrimer $\mathrm{AD}$ which was able to mediate safe and effective delivery of siRNA into primary microglia cultures. This dendrimer formed stable nanoparticles with siRNA, protected it 
from degradation and facilitated its uptake in microglia without affecting basal microglial properties. Dendrimerdelivered siRNA decreased target gene and protein expression, with consequent biological effects in a cellular model, which mimics the interactions between tumor cells and microglia within the brain tumor microenvironment. This dendrimer therefore constitutes a promising nanovector for siRNA delivery into microglia.

\section{Future perspective}

To our knowledge, this is the first report on dendrimer-mediated functional siRNA delivery to primary microglia without affecting the basal microglial functions and responses. As microglial cells are the principal immune cells of the brain and there is growing evidence to suggest that they have a role in many brain pathologies, the dendrimer $\mathrm{AD}$ presented in this work constitutes a promising innocuous carrier for siRNA delivery into microglia, and opens up new perspectives on functional genomic studies and therapeutic targeting of microglia in brain diseases. Further advances in RNAi technology, such as the design of better siRNA entities, combined with enhanced capabilities of AD to deliver siRNA specifically to microglia, may open up new possibilities for studies on microglial function in various brain pathologies and for the development of RNAi-based therapeutics. We are actively working in this direction.

\section{Summary points}

- Genetic manipulation of microglial functions in diseases using small interfering RNA (siRNA) has so far been hampered by the lack of efficient and harmless siRNA delivery methods.

- This is the first report on functional siRNA delivery, mediated by an amphiphilic dendrimer nanovector, into primary microglia with no effect on the basal microglial functions and responses.

- The amphiphilic dendrimer reported in this work is able to mediate safe and effective delivery of siRNA, and decrease targetgene and protein expression in primary microglia cultures.

- This dendrimer formed stable nanoparticles with siRNA, protected the siRNA from degradation, facilitated its cellular uptake and also enabled endosomal release.

- In contrast to the commercial nonviral vector or shRNA-carrying lentiviral vector, the dendrimer-mediated siRNA-delivery did not trigger inflammatory signaling responses in primary microglia.

- Microglia cells treated with dendrimer/siRNA complexes retained their ability to proliferate and respond to glioma cell-derived stimuli.

- Knockdown of the transcription regulator Id1 in microglia led to significant changes in gene expression, for example, the downregulation of cell cycle-related genes and upregulation of genes associated with the immune response.

- This dendrimer constitutes a promising innocuous carrier for siRNA delivery into microglia and opens up new perspectives on functional genomic studies and therapeutic targeting of microglia in brain diseases.

Supplementary data

To view the supplementary data that accompany this paper please visit the journal website at: www.futuremedicine.com/doi/full/10.2217/nnm-2019-0176

Financial \& competing interests disclosure

The authors are grateful for financial support from National Science Centre Poland (no. 2017/25/B/NZ3/02483 to AEM), La Ligue Nationale Contre le Cancer (EL2016.LNCC/LPP to LP), Italian Association for Cancer Research (AIRC, IG 17413, SP), the French National Research Agency under the frame of the Era-Net EURONANOMED European Research projects "Target4Cancer", "NANOGLIO" and "TARBRAINFECT" (LP), H2020 NMBP "SAFE-N-MEDTECH" (LP), European COST Action CA 17140 "Cancer Nanomedicine from the Bench to the Bedside", China Scholarship Council (LD) and Fondation pour la Recherche Médicale (SPF20160936294 to YJ). The authors have no other relevant affiliations or financial involvement with any organization or entity with a financial interest in or financial conflict with the subject matter or materials discussed in the manuscript apart from those disclosed.

No writing assistance was utilized in the production of this manuscript. 


\section{References}

Papers of special note have been highlighted as: $\bullet$ of interest; $\bullet \bullet$ of considerable interest

1. Li Q, Barres BA. Microglia and macrophages in brain homeostasis and disease. Nat. Rev. Immunol. 18, 225 (2017).

- Summarizes the versatile role of microglia in healthy and diseased brain.

2. Prinz M, Priller J. Microglia and brain macrophages in the molecular age: from origin to neuropsychiatric disease. Nat. Rev. Neurosci. 15, 300-312 (2014).

3. Graeber MB, Streit WJ. Microglia: biology and pathology. Acta Neuropathol. 119(1), 89-105 (2010).

4. Kaminska B, Mota M, Pizzi M. Signal transduction and epigenetic mechanisms in the control of microglia activation during neuroinflammation. Bioch. Biophys. Acta 1862(3), 339-351 (2015).

5. Ransohoff RM, El Khoury J. KNIHA - microglia in health and disease. Cold Spring Harb. Perspect. Biol. 8(1), a020560 (2015).

6. Ritzel RM, Patel AR, Grenier JM et al. Functional differences between microglia and monocytes after ischemic stroke. J. Neuroinflam. 12, 106 (2015).

7. Zhang B, Gaiteri C, Bodea L-G et al. Integrated systems approach identifies genetic nodes and networks in late-onset Alzheimer's disease. Cell 153(3), 707-720 (2013).

8. Mondelli V, Vernon AC, Turkheimer F et al. Brain microglia in psychiatric disorders. Lancet Psychiatry 4(7), 563-572 (2016).

9. Keren-Shaul H, Spinrad A, Weiner A et al. A unique microglia type associated with restricting development of Alzheimer's disease. Cell 169(7), 1276-1290 (2017).

10. Hambardzumyan D, Gutmann DH, Kettenmann H. The role of microglia and macrophages in glioma maintenance and progression. Nat. Neurosci. 19, 20-27 (2015).

- Provides a summary of the mechanisms of microglia and macrophage interaction within tumor microenvironment of malignant gliomas and opportunities for their therapeutic targeting.

11. Simmons GW, Pong WW, Emnett RJ et al. Neurofibromatosis-1 heterozygosity increases microglia in a spatially and temporally restricted pattern relevant to mouse optic glioma formation and growth. J. Neuropathol. Exp. Neurol. 70(1), 51-62 (2011).

12. Chen X, Zhang L, Zhang IY et al. RAGE expression in tumor-associated macrophages promotes angiogenesis in glioma. Cancer Res. 74(24), 7285-7297 (2014).

13. Gabrusiewicz K, Ellert-Miklaszewska A, Lipko M, Sielska M, Frankowska M, Kaminska B. Characteristics of the alternative phenotype of microglia/macrophages and its modulation in experimental gliomas. PLoS ONE 6(8), e23902 (2011).

-• Provides a rational for blockade of microglia/macrophage infiltration and their pro-invasive functions as a novel therapeutic strategy in malignant gliomas.

14. Poon CC, Sarkar S, Yong VW, Kelly JJP. Glioblastoma-associated microglia and macrophages: targets for therapies to improve prognosis. Brain 140(6), 1548-1560 (2017).

15. Setten RL, Rossi JJ, Han S-P. The current state and future directions of RNAi-based therapeutics. Nat. Rev. Drug Discov. 18(6), 421-446 (2019).

16. Wilson RC, Doudna JA. Molecular mechanisms of RNA interference. Annu. Rev. Biophys. 42(1), 217-239 (2013).

17. Hannon GJ. RNA interference. Nature 418(6894), 244-251 (2002).

18. Kanasty R, Dorkin JR, Vegas A, Anderson D. Delivery materials for siRNA therapeutics. Nat. Mater. 12(11), 967-977 (2013).

-• Describes the biological challenges that siRNA delivery materials aim to overcome, and the most effective and clinically advanced classes of siRNA delivery systems.

19. Whitehead KA, Langer R, Anderson DG. Knocking down barriers: advances in siRNA delivery. Nat. Rev. Drug Discov. 8(2), 129-138 (2009).

20. Rao S, Morales AA, Pearse DD. The comparative utility of viromer RED and lipofectamine for transient gene introduction into glial cells. BioMed Res. Int. 2015, 458624 (2015).

21. Butovsky O, Jedrychowski MP, Moore CS et al. Identification of a unique TGF- $\beta$-dependent molecular and functional signature in microglia. Nat. Neurosci. 17, 131 (2013).

22. Uemura $Y$, Naoi T, Kanai Y, Kobayashi K. The efficiency of lipid nanoparticles with an original cationic lipid as a siRNA delivery system for macrophages and dendritic cells. Pharm. Dev. Technol. 24(3), 263-268 (2018).

23. Zhang F, Nance E, Alnasser Y, Kannan R, Kannan S. Microglial migration and interactions with dendrimer nanoparticles are altered in the presence of neuroinflammation. J. Neuroinflam. 13(1), 65 (2016).

24. Cao Y, Liu X, Peng L. Molecular engineering of dendrimer nanovectors for siRNA delivery and gene silencing. Front. Chem. Sci. Eng. 11(4), 663-675 (2017).

25. Kesharwani P, Iyer AK. Recent advances in dendrimer-based nanovectors for tumor-targeted drug and gene delivery. Drug Discov. Today 20(5), 536-547 (2015). 
26. Wei T, Chen C, Liu J et al. Anticancer drug nanomicelles formed by self-assembling amphiphilic dendrimer to combat cancer drug resistance. Proc. Natl Acad. Sci. USA 112(10), 2978-2983 (2015).

27. Garrigue P, Tang J, Ding L et al. Self-assembling supramolecular dendrimer nanosystem for PET imaging of tumors. Proc. Natl Acad. Sci. 115(45), 11454-11459 (2018).

28. Dong Y, Yu T, Ding L et al. A dual targeting dendrimer-mediated siRNA delivery system for effective gene silencing in cancer therapy. $J$. Am. Chem. Soc. 140(47), 16264-16274 (2018).

-. This is the first report on the amphiphilic dendrimer for targeted delivery using a dual targeting peptide to decorate the siRNA/dendrimer nanoparticles for specific siRNA delivery in cancer cells within the tumor for more effective gene silencing and the resulting more potent anticancer activity.

29. Kesharwani P, Banerjee S, Gupta U et al. PAMAM dendrimers as promising nanocarriers for RNAi therapeutics. Mater. Today 18(10), 565-572 (2015).

30. Hsu HJ, Bugno J, Lee SR, Hong S. Dendrimer-based nanocarriers: a versatile platform for drug delivery. Wiley Interdiscip. Rev. Nanomed. Nanobiotechnol. 9(1), e1409 (2017).

31. Zhou J, Wu J, Hafdi N, Behr J-P, Erbacher P, Peng L. PAMAM dendrimers for efficient siRNA delivery and potent gene silencing. Chem. Comm. 22, 2362-2364 (2006).

32. Sonawane ND, Szoka FC, Verkman AS. Chloride accumulation and swelling in endosomes enhances DNA transfer by polyamine-DNA polyplexes. J. Biol. Chem. 278(45), 44826-44831 (2003).

33. Chen C, Posocco P, Liu X et al. Mastering dendrimer self-assembly for efficient siRNA delivery: from conceptual design to in vivo efficient gene silencing. Small 12(27), 3667-3676 (2016).

34. Liu X, Liu C, Zhou J et al. Promoting siRNA delivery via enhanced cellular uptake using an arginine-decorated amphiphilic dendrimer. Nanoscale 7(9), 3867-3875 (2015).

35. Liu X, Wang Y, Chen C et al. A fluorinated bola-amphiphilic dendrimer for on-demand delivery of siRNA, via specific response to reactive oxygen species. Adv. Function. Mater. 26(47), 8594-8603 (2016).

36. Liu X, Zhou J, Yu T et al. Adaptive amphiphilic dendrimer-based nanoassemblies as robust and versatile siRNA delivery systems. Angew. Chem. Int. Ed. 53(44), 11822-11827 (2014).

-• Describes the amphiphilic dendrimer for functional siRNA delivery in diverse cell types including primary cells, stems cells and immune cells such as T-cells and B-cells.

37. Yu T, Liu X, Bolcato-Bellemin A-LL et al. An amphiphilic dendrimer for effective delivery of small interfering RNA and gene silencing in vitro and in vivo. Angew. Chem. Int. Ed. 51(34), 8478-8484 (2012).

- $\quad$ Reports the first amphiphilic dendrimer for functional siRNA delivery in vitro and in vivo.

38. Lyu Z, Ding L, Huang AY-T, Kao C-L, Peng L. Poly(amidoamine) dendrimers: covalent and supramolecular synthesis. Mater. Today. Chem. 13, 34-48 (2019).

39. Walentynowicz KA, Ochocka N, Pasierbinska M et al. In search for reliable markers of glioma-induced polarization of microglia. Front. Immunol. 9(JUN), 1329 (2018).

40. Zawadzka M, Kaminska B. A novel mechanism of FK506-mediated neuroprotection: downregulation of cytokine expression in glial cells. Glia 49(1), 36-51 (2005).

41. Bolger AM, Lohse M, Usadel B. Trimmomatic: a flexible trimmer for Illumina sequence data. Bioinformatics 30(15), $2114-2120$ (2014).

42. Dobin A, Davis CA, Schlesinger F et al. RNA-STAR: ultrafast universal spliced sequences aligner: supplementary materials. Bioinformatics 29(1), 15-21 (2012).

43. Liao Y, Smyth GK, Shi W. featureCounts: an efficient general purpose program for assigning sequence reads to genomic features. Bioinformatics 30(7), 923-930 (2013).

44. Tarazona S, Furió-Tarí P, Turrà $\mathrm{D}$ et al. Data quality aware analysis of differential expression in RNA-seq with NOISeq R/Bioc package. Nucleic. Acids Res. 43(21), e140 (2015).

45. Yu G. clusterProfiler: an universal enrichment tool for functional and comparative study. bioRxiv doi:10.1101/256784 (2018).

46. Ellert-Miklaszewska A, Dabrowski M, Lipko M, Sliwa M, Maleszewska M, Kaminska B. Molecular definition of the pro-tumorigenic phenotype of glioma-activated microglia. Glia 61(7), 1178-1190 (2013).

- Describes the molecular pathways that direct microglia toward the pro-inflammatory or pro-invasive, immunosuppressive phenotype.

47. Wathelet MG, Lin CH, Parekh BS et al. Virus infection induces the assembly of coordinately activated transcription factors on the IFN- $\beta$ enhancer in vivo. Mol. Cell 1(4), 507-518 (1998).

48. Wendeln AC, Degenhardt K, Kaurani L et al. Innate immune memory in the brain shapes neurological disease hallmarks. Nature 556(7701), 332-338 (2018).

49. Ellert-Miklaszewska A, Wisniewski P, Kijewska M et al. Tumour-processed osteopontin and lactadherin drive the protumorigenic reprogramming of microglia and glioma progression. Oncogene 35(50), 6366-6377 (2016). 
50. Norton JD. ID helix-loop-helix proteins in cell growth, differentiation and tumorigenesis. J. Cell Sci. 113(22), 3897-3905 (2000).

51. Zebedee Z, Hara E. Id proteins in cell cycle control and cellular senescence. Oncogene 20(58), 8317-8325 (2001).

52. Jankovic V, Ciarrocchi A, Boccuni P, Deblasio T, Benezra R, Nimer SD. Id1 restrains myeloid commitment, maintaining the self-renewal capacity of hematopoietic stem cells. Proc. Natl Acad. Sci. 104(4), 1260-1265 (2007).

53. Papaspyridonos M, Matei I, Huang Y et al. Id1 suppresses anti-tumour immune responses and promotes tumour progression by impairing myeloid cell maturation. Nat. Commun. 6, 6840 (2015).

54. Mieczkowski J, Kocyk M, Nauman P et al. Down-regulation of IKK $\beta$ expression in glioma-infiltrating microglia/macrophages is associated with defective inflammatory/immune gene responses in glioblastoma. Oncotarget 6(32), 33077-33090 (2015).

55. Guo X, Wang H, Li Y et al. Transfection reagent lipofectamine triggers type I interferon signaling activation in macrophages. Immunol. Cell Biol. 97(1), 92-96 (2019). 